Journal of Engineering and Applied Sciences 14 (9): 3137-3143, 2019

ISSN: 1816-949X

(C) Medwell Journals, 2019

\title{
Control for Path Tracking of an AGV Robot
}

\author{
Kevin S. Sarmiento G., Oscar L. Laguna S., Javier E. Vasquez L., \\ Ruben D. Hernandez B. and Carolina Sandoval \\ Innovatic Research Group, Department of Mechatronics Engineering, \\ University Piloto of Colombia, Carrera 9 No. $45^{\text {a }}-44$ Bogota, Colombia
}

\begin{abstract}
This study presents the methodology used to develop a navigation system for autonomous robotic vehicles using fuzzy control techniques and the application of computational vision algorithms for tracking a particular mobile object in real time. The developed system consists of a robot of two wheels of differential traction of which its kinematic model is presented, controlled remotely through a closed loop of control. The computer vision technique is used for the perception of the environment through the camera embarked on the vehicle. The technique of fuzzy control is implemented in the system to interpret and make the decisions of action of the robot during navigation in a poorly structured environment in addition to the uncertainties associated to the tracking of the objective through the image. Through the validations made for the fuzzy control, the desired results are obtained that in this case corresponds to the monitoring of a given objective in real time.
\end{abstract}

$\underline{\text { Key words: } \text { Mobile robotics, directional control, path tracking, autonomous, application, implemented }}$

\section{INTRODUCTION}

The mobile robotic has evolved in diverse applications to satisfy the needs in the optimization time in determined tasks by means of the control, artificial intelligence among other, providing the support and the security in the execution of them (Bermudez 2002; Morin and Samson, 2008). The principal emphasis is on the operational problems known as displacement which involve the parameters of the path recognition and planning in complex unstructured environments.

Thus, ground robotic vehicles are distinguished mainly by their traction, steering and performance aspect which can be sub-actuated to completely non-holonomic, i.e. having less controllable degrees of freedom and which cannot be displaced laterally. According of parameters, the control systems are projected satisfying their physical and mechanics characteristics (Morin and Samson, 2008). However, the control of wheeled vehicles presents difficulties due to the non-holonomic constrains characteristics of the kinematic model and in addition to the saturation of the actuators. Some solutions to this problem are addressed by Kim and Oh (1998) and Kanayama et al. (1990). In the first of these, the reference speed of the robot can not be zero, therefore, it cannot perform position tasks. By Kim and Oh (1998) a stable control law is obtained, other approaches similar to this type of systems are obtained with fuzzy controller (Kanayama et al., 1990). The problem of the position stabilization means positioning at the final destination of the vehicle starting at any point with reference to the path, taking into account the location as the orientation with respect to the global coordinate systems.

In accordance with the above, a planning and execution of trajectories is made using with different methods and devices. The selected vehicle has similar characteristics to a real vehicle. Scale 1:10 used in professional skills of vehicle to scale, so, it is considered for mathematical analysis the Ackerman type which can be simplified to the bicycle model ( $a$ front wheel and rear wheel). This model allows explaining by equations based on geometric relation the physical properties of the vehicle (Garcia and Ximhai, 2005).

Once the equations that defined the system were obtained, they were implemented in Simulink of MATLABR with the objective of validating the behavior of vehicle and also analyzed the physical restrictions. When the validation of model was made the implementation and simulation of the steering control of the vehicle was continued considering the position and orientation variables supplied by the simulated GPS. This

Corresponding Author: Kevin S. Sarmiento G., Innovatic Research Group, Department of Mechatronics Engineering, University Piloto of Colombia, Carrera 9 No. $45^{\text {a }}-44$ Bogota, Colombia 


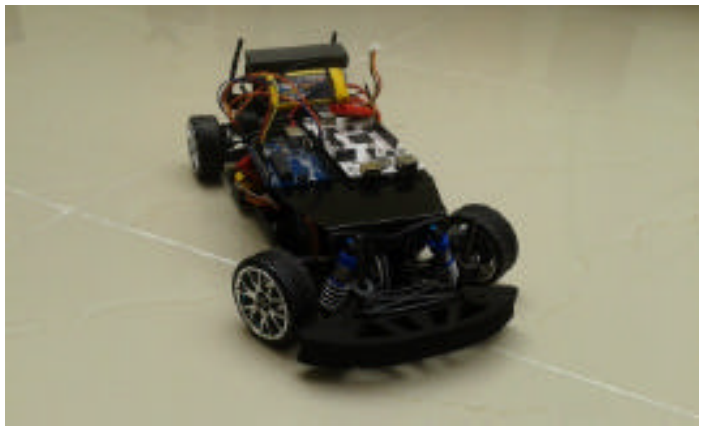

Fig. 1: Platform adopted

algorithm allows moving the robot to a certain point of the system passing through a previously defined path.

In the scenario in which the movement will be executed, a camera was placed at a distance of $4 \mathrm{~m}$ above the ground taking the area of execution of the trajectory, by means of filters of color detection and centroid positioning the simulation of a GPS was implemented which determines the orientation and position of the vehicle within the research area in which the trajectory tracking will be executed.

Mechanical structure: The platform used for this project is based on a small vehicle scale (1:10) and with approximate characteristics to a commercial vehicle.

The 1/10th scale RC 4WD high performance CAR carriage (Fig. 1) was implemented since its mechanical structure meets the functional and kinematic requirements required to perform trajectory tracking tests in a predetermined environment.

This vehicle has a differential system (4-wheel drive) and its dimensions are appropriate to carry out all the electronic assembly necessary for the functional development of the process. In addition, the steering system of the vehicle is based on the Ackerman Model, through the geometry of this model the front wheels rotate with different angles. Figure 2 presents a sketch of two types of systems, the first one (Fig. 2a) shows a schematic with the axis arm at a $90^{\circ}$ angle and the other schematic (Fig. 2b) following the Ackerman Model with the shaft arm with a $105^{\circ}$ angle. The $90^{\circ}$ model makes the 2 front wheels turn parallel with the same angle and its main disadvantage is the loss of performance and slip at the time of the curve. Otherwise, in the Ackerman Model, it ensures that the steering angles of the front wheels are different at the time of making the curve as shown in Fig. $2 b$.

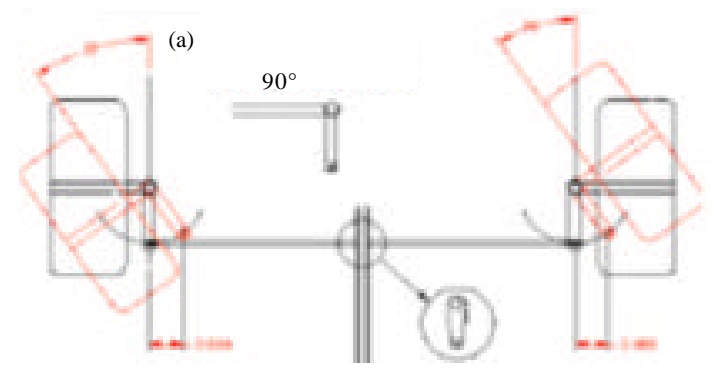

(b)

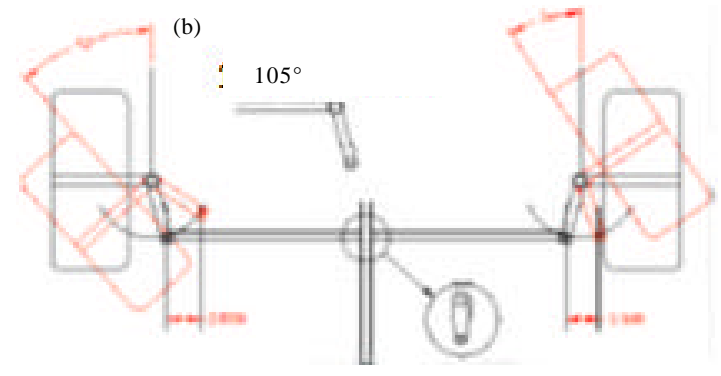

Fig. 2: Configuration of the steering system: a) Conventional direction and b) Ackerman direction

\section{MATERIALS AND METHODS}

Mathematical model: The mathematical model allows the physical properties of the vehicle to be explained by equations based on geometric relations without considering the forces involved in its movement (Garcia and Ximhai, 2005). In the first instance, a two-dimensional Cartesian global coordinate system is established that is represented in the horizontal plane $(\mathrm{x}, \mathrm{y})$ in which the parameters used in Eq. 1 are taken into account:

$$
X=\left[\begin{array}{l}
x \\
y \\
\theta
\end{array}\right]
$$

The identification in Cartesian space is given by the coordinates $(x, y)$ and the overall orientation of the vehicle is represented by $\theta$.

Kinematic Model: The kinematic model is based on the Ackerman Model, the advantage of this model is that it does not consider the slippage of the tires under certain conditions at low speeds (Carames, 2012.). Considering that it is assumed that the system has two rear wheels with a fixed axle and two front wheels which are operated by a bar to control the steering (Fig. 2). The characteristic components of this model are defined by a steering angle $\left(\delta_{0}\right)\left(\delta_{i}\right)$ an orientation angle $(\theta)$ the longitudinal separation between the wheels (L) the lateral spacing between the wheels (b) and the point of the vehicle centre of gravity $\left(X_{c} Y_{c}\right)$, relative to a global reference system $(\mathrm{X}, \mathrm{Y})$ as a show in Fig. 3. 


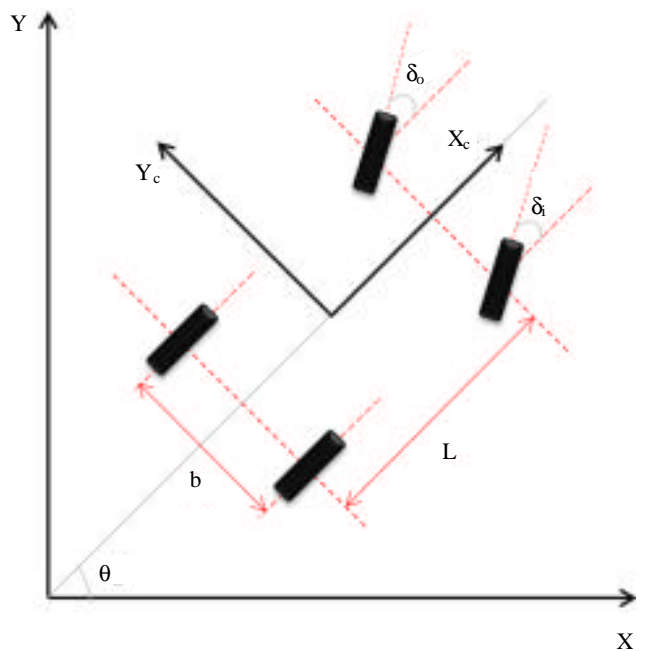

Fig. 3: Ackerman configuration

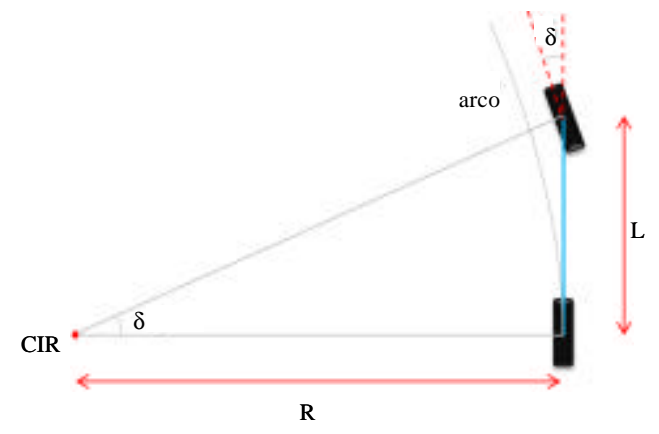

Fig. 4: Geometry of the bicycle model

Given that the vehicle operates at low speeds, this mathematical model can be simplified with the bicycle model (Oscar and Duran, 2015) which would later allow the simulation for trajectory tracking. The model of the bicycle considers that the four wheels of the vehicle can be replaced by only two (a rear wheel and a front wheel), just as the steering angles of the front wheels are replaced at a single angle and the point of centre of gravity of the vehicle.

This model reduces geometric relationships and approximates the characteristics of the Ackerman Model at reasonably low speeds with moderate steering angles (Ejarque et al., 2008) (Fig. 4). Thus, a simplified model describing the IRC (Instantaneous Rotation Centre) of the robot can be observed taking into account the previously mentioned reductions. From the model, the following geometric relation is obtained:

$$
\operatorname{Tan}_{\delta}=\frac{\mathrm{L}}{\mathrm{R}}
$$

where, it is possible to determine the radius of curvature of the vehicle, dependent on the value of the steering angle $(\delta)$ as seen in Fig. 5.

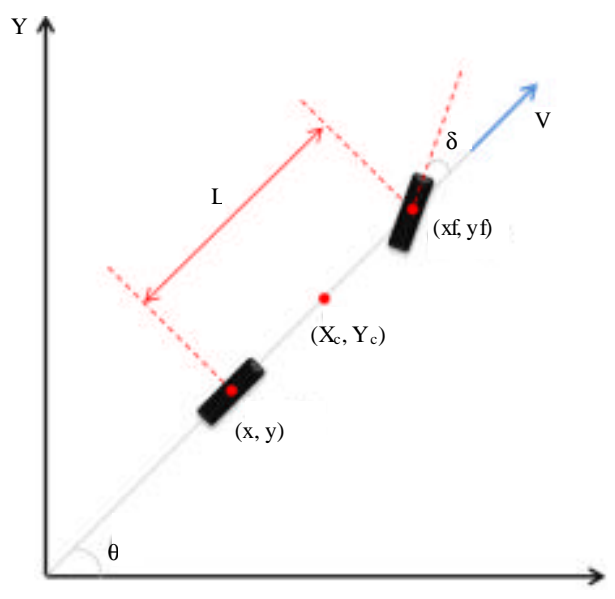

Fig. 5: Kinematic Model of the bicycle

To define the characteristic equations of the vehicle, it is necessary to determine the non-holonomic constraints for the front and rear wheels (Luca et al., 1998; Rueda and Miguel, 2013):

$$
\begin{gathered}
\dot{x} f \operatorname{sen}(\theta+\delta)-\dot{y} f \cos (\theta+\delta)=0 \\
\dot{x} \operatorname{sen}(\theta)-\dot{y} \cos (\theta)=0
\end{gathered}
$$

For which it is taken into account that $(x, y)$ defines the global coordinates of the rear wheel and $(\mathrm{xf}, \mathrm{yf})$ the coordinates of the front wheel. It is also possible to express the overall coordinates of the front wheel as follows:

$$
\begin{aligned}
& x f=x+L \cos (\theta) \\
& y f=y+L \operatorname{sen}(\theta)
\end{aligned}
$$

From Eq. 5 and 6 in Eq. 3 and taking into account that Eq. 4 is satisfied with $\dot{x}=\cos (\theta)$ and $\dot{y}=\operatorname{sen}(\theta)$ any scalar thereof is evaluated for the corresponding vehicle velocity (Corke, 2011), so that, the kinematic equations of the system can be expressed as:

$$
\begin{aligned}
& \dot{x}=v \cos (\theta) \\
& \dot{y}=v \operatorname{sen}(\theta) \\
& \dot{\theta}=\frac{v \tan (\delta)}{L}
\end{aligned}
$$

Proposed system: The communication process to make effective the development of the trajectory is given through the interaction of an IP camera, a computer and 
an actuation system, backed up by the simulations previously carried out. The aerial camera supplies the information of Latitude, longitude and orientation of the vehicle in order to locate it within the coordinate plane of research to achieve this development the camera sends frames per second to the computer and there through the use of Qt Software are characterized such parameters by the detection of colors. Also within the computer is the development of the algorithm of direction control.

Once the positions of the robot have been characterized and the algorithm has been implemented, the signals of both speed and direction are sent to the vehicle by means of Bluetooth communication to the electronic system. For this, the vehicle will execute the defined trajectory, adjusting the direction, taking into account the signals coming from the computer until realizing the total execution of the movement (Fig. 6).

The planning of the trajectory: The planning of the trajectory was defined point to point, however, to realize this trajectory it is necessary to keep in mind the dimensions of the work area, captured by the installed aerial camera. Considering of this parameter a path is defined considering the characteristic equations of the

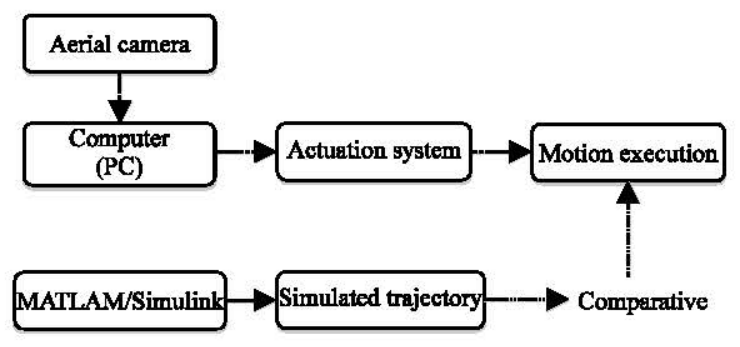

Fig. 6: Systems architecture system which was developed taking into account a constant speed and sent pulses to change the direction sense in a determined time instant, that is to say, left and right turn which visually in the xy plane is observed as a lane change.

Obtained the ideal path, a series of points are defined, so, the path is defined as the successive composition of a series of points by which the vehicle will have to pass in a certain order determined.

The vehicle will have a position and initial orientation, once defined the sequentially of the points that will conform the trajectory, the robot should automatically recognize the first point to be addressed, arriving at the coordinates of the first point planning the next and to define the simulated ideal trajectory.

Algorithm developed: The implementation of the algorithm aims to orient the vehicle to the desired position given the points that define the trajectory and the initial conditions of the system (Corke, 2011; Hossen et al., 2017). In the first instance is considered the speed as constant for the vehicle during the execution of its movement. It starts from the problem that the robot will have to reach a point (p) defined in the coordinate system of research (Fig. 7):

$$
\mathrm{p}=\left(\mathrm{x}^{*} \mathrm{y}^{*}\right)
$$

To move the vehicle to the point indicated taking into account initial and final coordinates is considering the overall orientation of the current system with respect to the desired system defined by:

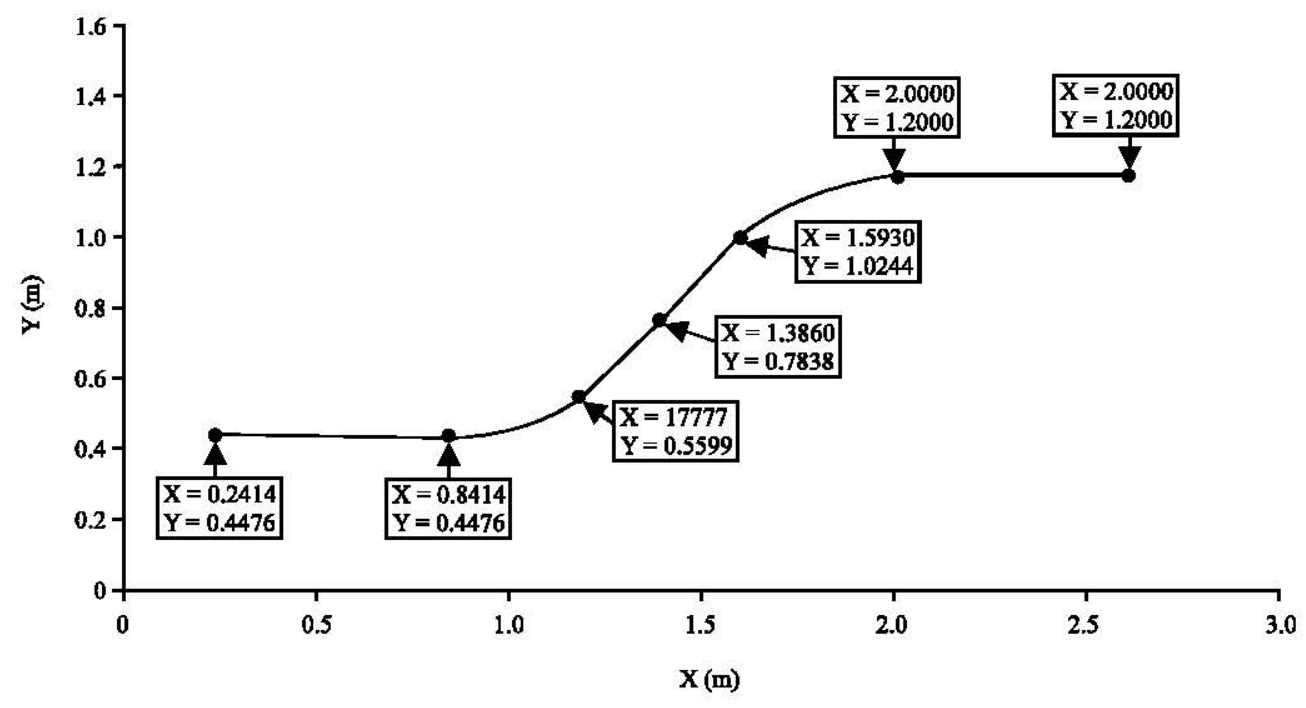

Fig. 7: Simulated trajectory and define point 


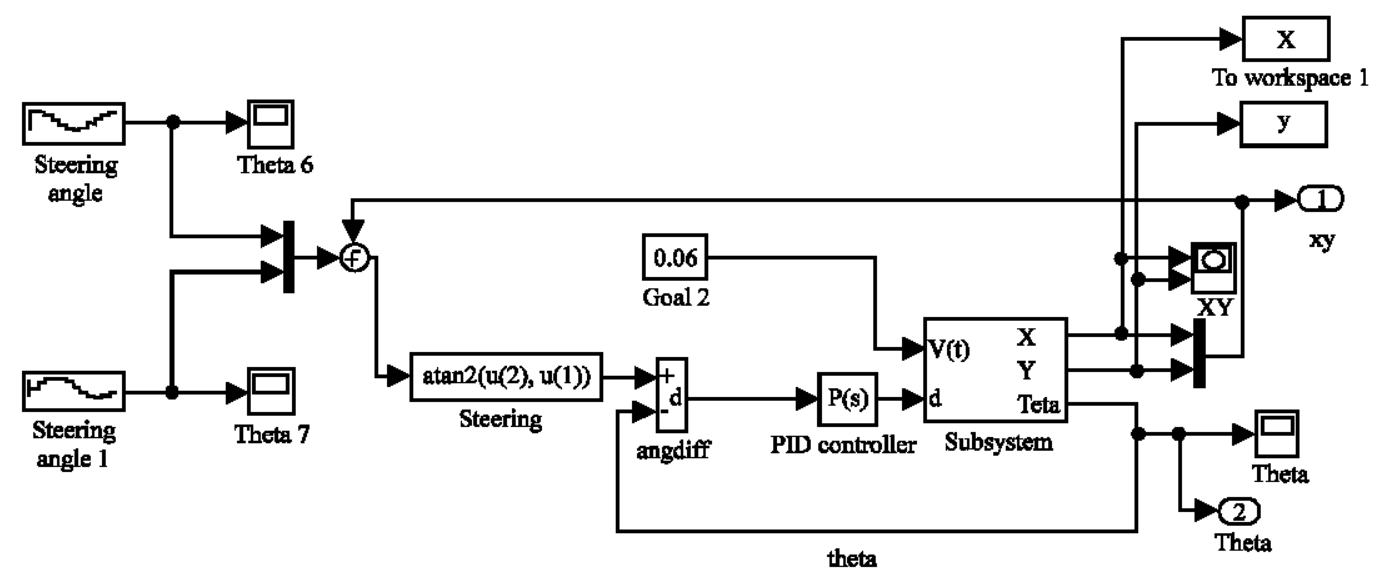

Fig. 8: Algorithm implemented in Simulink-MATLAB $\mathbb{R}$

$$
\theta^{*}=\tan ^{-1} \frac{y^{*}-y}{x^{*}-x}
$$

The next consideration is to evaluate one of the characteristics equations of the system previously determinate:

$$
\dot{\theta}=\frac{\operatorname{vtan}(\delta)}{L}
$$

The steering control lies in realizing the difference between the angular $\theta^{*}$ and $\theta$ by returning a difference in the interval between $(-\pi, \pi)$ (Corke, 2011), the vehicle adjusts the direction of the front wheels in the order to reach the desired coordinate.

To reduce the error and improve the system response, a proportional controller was implemented in the value of the angular correction defined by:

$$
\delta=\mathrm{K}_{\mathrm{h}} * \theta^{*}
$$

Begin $\mathrm{K}_{\mathrm{h}}>0$. Where, $\theta^{*}$ represents the diretion correct value of the interval between $(-\pi, \pi)$. Similarly, it is necessary to consider that the vehicle has a mechanical limitation in its direction by such that it establishes that the front wheels have a maximum (external) rotation of $n$ degrees with respect to its center, therefore, it is considered that:

$$
\begin{aligned}
& \text { Right tire }=\delta_{\max } \\
& \text { Left tire }=-\delta_{\max }
\end{aligned}
$$

According to the mentioned parameters, the whole system is developed in Simulink of MATLABß to aim simulate the behavior of the vehicle in the execution of the trajectory (Fig. 8).

To implement the algorithm in the vehicle was used the free Software Qt in the Linux operating system which is a library used to develop applications in $\mathrm{C} \mathrm{+H}$ graphical interface. In this application, the code was implemented in which the computational vision development was applied to perform of the color identification (simulated GPS), obtaining data and communication with the electronic system boarded in the vehicle.

\section{RESULTS AND DISCUSSION}

For the defined trajectory, it can be observed that the response of the vehicle seeks to minimize the steering error in order to follow the desired trajectory. The gain of the proportional controller is $\mathrm{K}_{\mathrm{p}}=4$ which satisfies that the steady error is $0.1 \%$, the result obtained is shown in Fig. 9.

Figure 10 shows the comparison of the desired trajectory (blue color) versus the resultant one by means of the implemented control (red color). As can be seen, the vehicle defines a large part of the trajectory and by means of the gain adjustments the orientation and reference errors are minimized.

In the same way, the graphical interface developed in Qt is obtained which allows observing the data of the working environment and the route of the vehicle while making the definition of the desired trajectory (Fig. 11).

The trajectory made by the vehicle in real time is shows in Fig. 12. The red point described in the image lower represents the starting point and the green points are the previously selected coordinates. These points are of reference that is to say, they are of visual support, so that, it is possible to observe the form of the road and to verify the execution of the trajectory. 


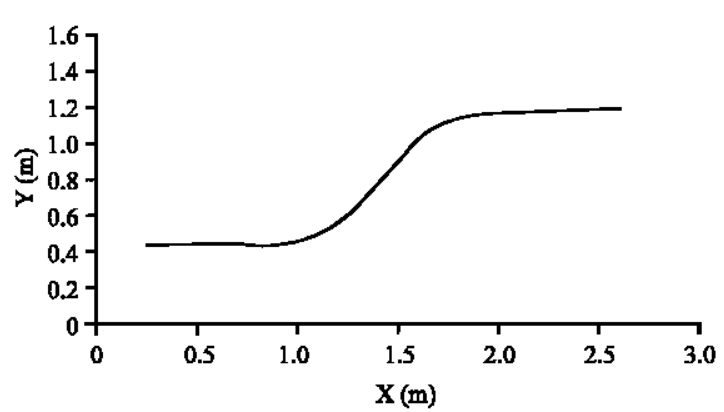

Fig. 9: Vehicle real trajectory

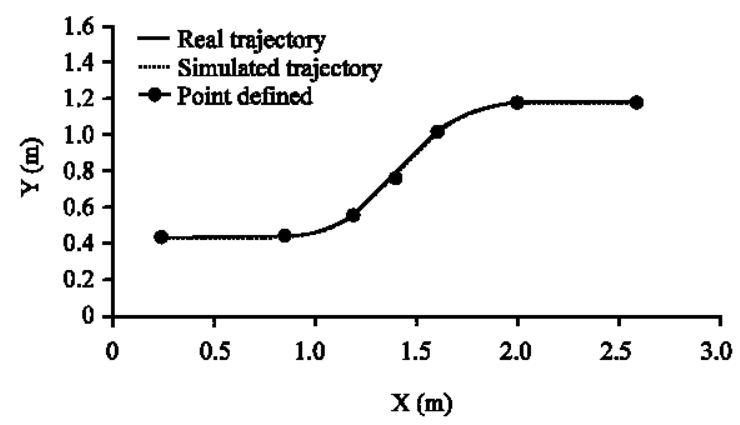

Fig. 10: Comparative real trajectory versus simulated

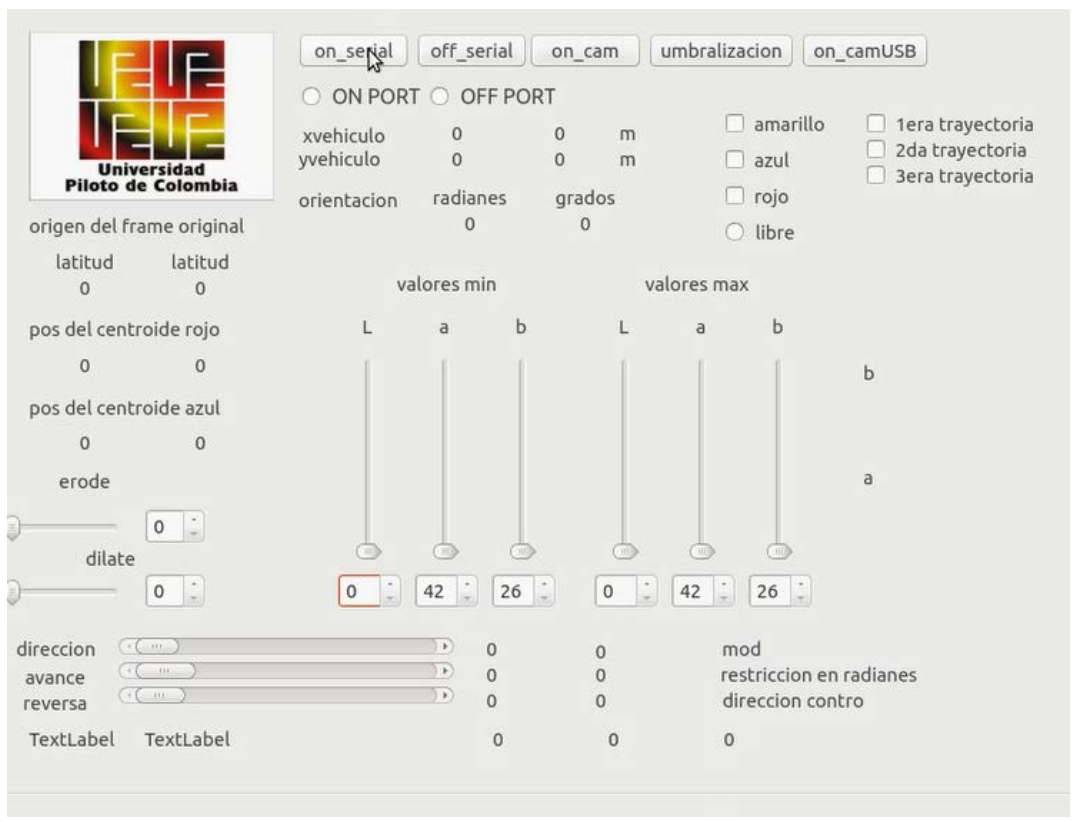

Fig. 11: Developed interphase

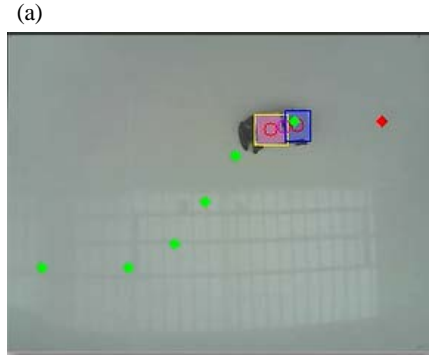

(b)
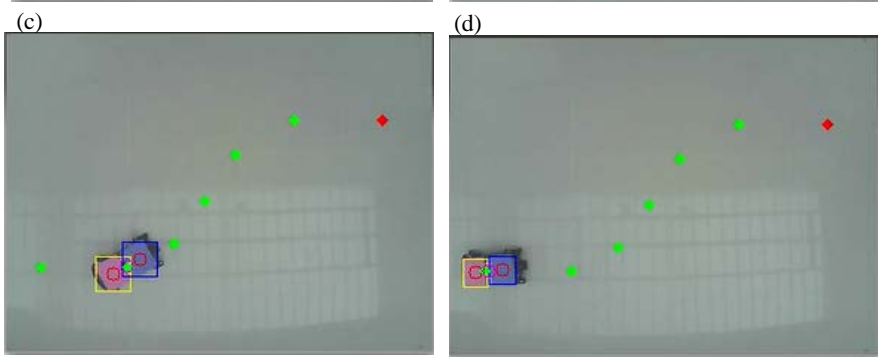

Fig. 12: a-d) Performance of the trajectory in real time 


\section{CONCLUSION}

The research presents the development of a vehicle in a scale to able to navigate independently to pre-established points defining a desired trajectory. This implementation was achieved through applications of computer vision, programming in $\mathrm{C}++$ and implementation of electronic devices.

The results of the simulations were truthful and approximate to reality due to the effective development of the mathematical model of the vehicle which allowed taking into account a series of parameters essential for the definition of the trajectory and execution of the movement.

The algorithm was implemented correctly, since, the vehicle presented a reasonable behavior according to the simulations of the proposed path. The application of this algorithm is valid and efficient for low speeds compared to high speeds, since, when adopting wireless communication there is a delay in the signal which would prevent a correct reading and execution of the movement at these speeds.

\section{ACKNOWLEDGEMENT}

This research was supported by the INNOVATIC Group Research in the Department of Mechatronics Engineering of Piloto de Colombia in Bogota, Colombia.

\section{REFERENCES}

Bermudez, G., 2002. [Mobile robots: Theory, applications and experiences (In Spanish)]. Mag. Tecnura, 5: 6-17.

Carames, C.F., 2012. [Navigation techniques for a mobile robot using spatial reasoning systems]. Ph.D Thesis, University of Salamanca, Salamanca, Spain. (In Spanish)

Corke, P., 2011. Robotics, Vision and Control: Fundamental Algorithms in Matlab. Vol. 73, Springer, Berlin, Germany,.
Ejarque, G., R. Haart and M. Distefano, 2008. [Mechanism of control of rotation of a spherical wheel]. Master Thesis, Institute of Automatic and Industrial Electronics (IAEI). National University of Cuyo, Mendoza, Argentina. (In Spanish)

Garcia, E.M. and R. Ximhai, 2005. Planning and tracking mobile robot trajectories in a simulation of a real environment. Master Thesis, Indigenous Autonomous University of Mexico, El Fuerte, Sinaloa, Mexico.

Hossen, J., S. Sayeed, T. Bhuvaneswari, C. Venkataseshaiah and J. Emerson et al., 2017. An automated fuzzy logic based low cost floor cleaning mobile robot. J. Eng. Appl. Sci., 12: 119-126.

Kanayama, Y., Y. Kimura, F. Miyazaki and T. Noguchi, 1990. A stable tracking control method for an autonomous mobile robot. Proceedings of the IEEE International Conference on Robotics and Automation, May 13-18, 1990, IEEE, Cincinnati, Ohio, ISBN:0-8186-9061-5, pp: 384-389.

Kim, D.H. and J.H. Oh, 1998. Globally asymptotically stable tracking control of mobile robots. Proceedings of the 1998 IEEE International Conference on Control Applications Vol. 2, September 4, 1998, IEEE, Trieste, Italy, ISBN:0-7803-4104-X, pp: 1297-1301.

Luca, D.A., G. Oriolo and C. Samson, 1998. Feedback Control of a Nonholonomic Car-Like Robot. In: Robot Motion planning and Control, Laumond, J.P. (Ed.). Springer, Berlin, Germany, ISBN:978-3-540-76219-5, pp: 171-253.

Morin, P. and C. Samson, 2008. Motion Control of Wheeled Mobile Robots. In: Springer Handbook of Robotics, Siciliano, B. and K. Oussama (Eds.). Springer, Berlin, Germany, ISBN:978-3-540-23957-4, pp: $799-826$.

Oscar, C. and E. Duran, 2015. [Locating an ackerman configuration mobile robot using the extended kalman filter]. Master Thesis, National University of Engineering, Lima, Peru. (In Spanish)

Rueda, C. and A. Miguel, 2013. [Hybrid localization for a stand-alone vehicle on scale using sensor fusion: Hybrid localization for an R/C car scale using sensor fusion]. Master Thesis, University of Campinas, Campinas, Brazil. (In Portugese) 